\title{
Higgs pair production in the NMSSM at the LHC
}

\author{
Ulrich Ellwanger \\ Laboratoire de Physique Théorique, UMR 8627, CNRS and \\ Université de Paris-Sud, \\ F-91405 Orsay, France \\ E-mail: ulrich.ellwanger@th.u-psud.fr
}

\begin{abstract}
In the NMSSM it is well possible to find an additional Higgs boson with a mass below $125 \mathrm{GeV}$ which remains invisible in standard Higgs boson search channels. We study the Higgs pair production cross sections times branching fractions in this scenario, focussing on gluon fusion and the $b \bar{b}+\tau^{+} \tau^{-}$and $b \bar{b}+\gamma \gamma$ final states. Summing over the SM-like and the lighter Higgs states, the production cross sections times branching fractions are never below the ones for SM Higgs pair production. Sizeable enhancements of the signal rates are also possible, notably if a lighter Higgs state is produced. However, the rates involving at least one lighter Higgs boson are not always sufficiently large to guarantee its discovery.
\end{abstract}

KeYWords: Supersymmetry Phenomenology

ArXiv ePrint: 1306.5541 


\section{Contents}

1 Introduction 1

2 Calculations of Higgs pair production cross sections in the NMSSM 3

3 Results for Higgs pair production cross sections in the NMSSM 4

4 Conclusions $\quad 9$

\section{Introduction}

The couplings of the $125 \mathrm{GeV}$ Higgs boson to electroweak gauge bosons, third generation fermions and the loop induced couplings to gluons and photons have been measured by the ATLAS and CMS collaborations with an astonishing precision already after the $7-8 \mathrm{TeV}$ runs $[1,2]$. Clearly, these measurements impose constraints on models with extended Higgs sectors.

However, extended Higgs sectors can manifest themselves mainly through deviations of Higgs self couplings from the values within the Standard Model (SM), which can now be determined once the Higgs mass is known. For this reason, measurements of Higgs self couplings (actually only the trilinear self coupling seems accessible in the foreseeable future) at the LHC at $14 \mathrm{TeV}$ at high integrated luminosity and/or at future colliders are of utmost importance.

The trilinear Higgs self coupling contributes to Higgs pair production which allows, in principle, its measurement. Many corresponding studies have been performed in the SM and its supersymmetric (Susy) extensions, notably at hadron colliders and considering gluon fusion [3-28], the production mode with the largest cross section.

Among the Susy extensions of the SM, the Next-to-Minimal Supersymmetric Standard Model (NMSSM) [29] has received considerable attention [30-52], since a Higgs mass of $125 \mathrm{GeV}$ is more natural within its parameter space than in the MSSM. Due to the additional gauge singlet superfield $S$ compared to the MSSM, the NMSSM contains three neutral CP-even Higgs states $H_{i}, i=1,2,3$ (ordered in mass).

It is quite natural in the parameter space of the NMSSM that the mostly SM-like state $H_{\mathrm{SM}}$ near $125 \mathrm{GeV}$ is actually the second lightest state $H_{2}$, wheras the lightest state $H_{1}$ is mostly singlet-like; then mixing effects contribute to the increase of the mass of $H_{\mathrm{SM}}$. A lighter mostly singlet-like state $H_{1}$ can well be compatible with the constraints from LEP [53] (simultaneously with a SM-like state near $125 \mathrm{GeV}$ [43]), and might even explain the mild $(\sim 2 \sigma)$ excess in $e^{+} e^{-} \rightarrow Z b \bar{b}$ near $M_{b \bar{b}} \sim 100 \mathrm{GeV}[53]$.

The pair production of two states $H_{\mathrm{SM}}$ in the NMSSM has been considered recently in $[26,28]$. In [26] it was found that, in the presence of light stops present in the loopinduced amplitudes, the production rate can be considerably larger than in the SM. In [28] it 
was emphasized that loop corrections to the trilinear Higgs couplings and hence to the pair production of two states $H_{\mathrm{SM}}$ can potentially be large. (The range of the trilinear Higgs couplings within some regions of the parameter space of the NMSSM have been studied in [54].)

In the present paper we concentrate on the case where the mostly SM-like state $H_{\mathrm{SM}}$ near $125 \mathrm{GeV}$ corresponds to $H_{2}$, and study the prospects of $H_{2}+H_{2}, H_{2}+H_{1}$ and $H_{1}+H_{1}$ pair production. Notably in the case of a small non-singlet component of $H_{1}, H_{1}$ will hardly couple to electroweak gauge bosons and fermions, and be practically invisible in standard Higgs search channels. However, the NMSSM-specific $H_{1} H_{2} H_{2}$ and/or $H_{1} H_{1} H_{2}$ couplings can still be large. These would allow to detect $H_{1}$ in $H_{2} \rightarrow H_{1}+H_{1}$ decays if $M_{H_{1}} \lesssim M_{H_{2}} / 2$. For $M_{H_{1}} \gtrsim M_{H_{2}} / 2$, the case considered here, Higgs pair production might be the only way to observe the $H_{1}$ state.

In principle $H_{1}$ could also be produced in $H_{3}$ decays $[49,55]$. However, this strategy would also fail if $H_{3}$ is too heavy. Subsequently we make the pessimistic assumption that this is the case, and that $H_{3}$ does not contribute to the Higgs pair production cross section. Likewise we assume that stops are too heavy to affect the Higgs pair production cross section; otherwise stops are likely to be discovered before the observation of Higgs pair production processes. Also light pseudoscalars are assumed to be absent. If the assumptions of a heavy $\mathrm{H}_{3}$, heavy pseudoscalars and heavy stops turn out to be wrong, we would be pleased to redo the calculations with correspondingly known masses.

At present the most promising search strategy for Higgs pair production at the LHC at $14 \mathrm{TeV}$ seems the application of subjet-based analysis techniques to boosted kinematical regimes of the dihiggs system [19]. According to [19], the $b \bar{b}+\tau^{+} \tau^{-}$final state seems accessible by this method. In the case of the NMSSM, the branching fractions into $b \bar{b}$ and $\tau^{+} \tau^{-}$can differ from the SM. Notably the lighter state $H_{1}$ will hardly decay into electroweak gauge bosons $W^{ \pm}$and $Z$, which implies a somewhat larger branching fraction into $b \bar{b}$ (and $\tau^{+} \tau^{-}$) than the $\sim 60 \%$ of a SM-like Higgs boson. The corresponding branching fractions of $\mathrm{H}_{2}$ can well be reduced. For these reasons we will study the Higgs pair production cross sections multiplied by the corresponding branching fractions normalized to the SM.

The $b \bar{b}+W^{+} W^{-}$final state in boosted kinematical regimes has been analysed as well using jet substructure techniques [21]. However, due to the reduced branching fractions of $H_{1}$ into $W^{+} W^{-}$we will not consider this channel here.

Another possible final state is $b \bar{b}+\gamma \gamma[17]$. In spite of the limited statistics, Higgs pair production may be observable in this channel if fake $b$-jets and photons are sufficiently under control. Hence we extend our analysis to this final state, again multiplying the Higgs pair production cross sections by the corresponding branching fractions normalized to the SM.

In the case of the state $H_{2}$ near $125 \mathrm{GeV}$, the measured couplings [1, 2] imply bounds on possible deviations from the SM. Here we will apply bounds (at the 95\% confidence level) on all couplings from a recent combination of the ATLAS, CMS and Tevatron results [56]. These constrain not only the $H_{2}$ branching fractions and couplings, but all NMSSM specific parameters.

In the next section we describe our calculations, in section 4 our results. Conclusions are devoted to section 3 . 


\section{Calculations of Higgs pair production cross sections in the NMSSM}

In any model for the Higgs sector, the effective potential describes the Higgs vacuum expectation values (vevs), the (running) Higgs masses and Higgs self couplings. Radiative corrections to the effective potential will affect all these quantities simultaneously. Given the knowledge of the values of the $\mathrm{SU}(2) \times \mathrm{U}(1)$ symmetry breaking vev and the SM-like Higgs mass, it is crucial to compute the Higgs self couplings at the same level of precision as the SM-like Higgs mass.

For a given set of input parameters (see below), the radiative corrections to the Higgs masses and couplings in the NMSSM can be sizeable [28, 29]. For not too light Higgs states circulating in the loops (as considered here), the dominant radiative corrections to the effective potential originate from stop/top quark loops. Since we scan over the input parameters of the general $\mathbb{Z}_{3}$-invariant NMSSM (and, to some extent, radiative corrections to couplings can be absorbed by modifications of the input parameters such that the SM-like Higgs mass remains unchanged) we confine ourselves to the dominant radiative corrections from stop/top quark loops to masses and Higgs self couplings in the leading logarithmic approximation. To this end we employ a correspondingly modified version of the code NMSSMTools [57-59].

The field content of the Higgs sector of the NMSSM are two SU(2) doublets $H_{u}, H_{d}$, and a gauge singlet $S$. The parameters in the Higgs sector of the general $\mathbb{Z}_{3}$-invariant NMSSM include two dimensionless Yukawa couplings $\lambda$ (proportional to a term $S H_{u} H_{d}$ in the superpotential) and $\kappa$ (a trilinear singlet self coupling in the superpotential), corresponding soft Susy breaking terms $A_{\lambda}, A_{\kappa}$, and three soft Susy breaking mass terms for $H_{u}, H_{d}$, and $S$. The three soft Susy breaking mass terms can be traded for the known value of $M_{Z}$ and the two variables $\tan \beta \equiv\left\langle H_{u}\right\rangle /\left\langle H_{d}\right\rangle, \mu_{\mathrm{eff}} \equiv \lambda\langle S\rangle$ (for more details see [29]). Hence we are left with the free variables

$$
\lambda, \kappa, A_{\lambda}, A_{\kappa}, \tan \beta \text { and } \mu_{\mathrm{eff}} .
$$

A mass of about $125 \mathrm{GeV}$ of a mostly SM-like Higgs boson $H_{2}$ is obtained naturally for larger values of $\lambda$ and relatively low values of $\tan \beta$. The results below are obtained from a scan over these parameters in the ranges

$$
\begin{aligned}
\lambda & =0.5-0.7, & \kappa & =0.07-0.5, \\
A_{\lambda} & =300-1000 \mathrm{GeV}, & A_{\kappa} & =-500-0 \mathrm{GeV}, \\
\tan \beta & =1.5-6, & \mu_{\mathrm{eff}} & =120-300 \mathrm{GeV} .
\end{aligned}
$$

Since we confine ourselves to the leading stop/top quark induced radiative corrections to the Higgs potential, we only have to specify the stop masses and mixing parameter. We choose $1 \mathrm{TeV}$ for the stop masses (allowing to safely neglect stop contributions to Higgs production in gluon fusion) and $A_{\text {top }}=0$ for the mixing parameter. (Large values for $A_{\text {top }}$ are not necessary in order to obtain a mostly SM-like Higgs boson at $125 \mathrm{GeV}$ through radiative corrections.) 
The Higgs spectrum in the above range of parameters contains no light CP-odd states. Together with the relatively low values of $\tan \beta$, the constraints from $\mathcal{B}$-physics implemented in NMSSMTools are always satisfied. More relevant are the constraints we impose on the CP-even Higgs sector.

First, we require a CP-even Higgs boson $\mathrm{H}_{2}$ of a mass of $125.7 \pm 2 \mathrm{GeV}$. Next we require that the signal rates of this state in the production modes via gluon fusion, vector boson fusion and in association with electroweak gauge bosons, and in the decay channels into two photons, electroweak gauge bosons, pairs of $b$-quarks and $\tau$-leptons are within the $95 \%$ confidence limits obtained in [56] from a combination of the measurements at ATLAS, CMS and the Tevatron. (In the NMSSM at low $\tan \beta$ one can safely assume that the Higgs couplings to $b$-quarks and $\tau$-leptons are rescaled by the same amount with respect to the $\mathrm{SM}$, so that the corresponding measurements can be combined.)

These bounds imply that, within the above parameter space of the NMSSM, the couplings of the Higgs boson near $125 \mathrm{GeV}$ to electroweak gauge bosons and fermions cannot deviate dramatically from the SM values, just the loop-induced coupling to photons can be significantly larger (depending on the production mode, see [56]).

On the mass of the lightest CP-even Higgs boson $H_{1}$ we impose $M_{H_{1}}>65 \mathrm{GeV}$. Otherwise $H_{2} \rightarrow H_{1}+H_{1}$ decays (with $H_{2}$ nearly on shell) would be possible with much larger signal rates than the Higgs pair production considered here, allowing to detect $H_{1}$ in single $\mathrm{H}_{2}$ production channels. Subsequently we assume that such on-shell Higgs-to-Higgs decays will not be observed.

The code NMSSMTools provides us with all required parameters (including the trilinear Higgs self couplings) for the calculation of the Higgs pair production cross sections in gluon fusion of all possible combinations of $H_{1}$ and $H_{2}$ states. For the calculation of the Higgs pair production cross sections we use the public code HPAIR [60] which includes QCD corrections and a low energy theorem for the top quark loop. We modified this code according to our needs and verified that, in cases where $H_{1}$ or $H_{2}$ are SM-like and the other state is omitted, a SM-like pair production cross section is obtained. (For a $125 \mathrm{GeV}$ SM Higgs boson, HPAIR gives a production cross section of about $32.6 \mathrm{fb}$ in gluon fusion at $14 \mathrm{TeV}$ c. m. energy.)

\section{Results for Higgs pair production cross sections in the NMSSM}

First we show our results for the trilinear Higgs couplings obtained from NMSSMTools from the scan described in section 2. These are defined in the convention where the the trilinear terms in the effective potential are written as

$$
V_{\mathrm{eff}}=\frac{1}{3 !} \sum_{i, j, k} g\left(H_{i}, H_{j}, H_{k}\right) H_{i} H_{j} H_{k}+\ldots
$$

in which the trilinear Higgs coupling of the SM has a value of about $190 \mathrm{GeV}$. Our subsequent results will be shown as function of $M_{H_{1}}$ which we study in the range $M_{H_{1}} \sim$ $65-125 \mathrm{GeV}$ (always below the SM-like $M_{H_{2}}$ ). In figure 1 we show scatter plots of $g\left(H_{2}, H_{2}, H_{2}\right), g\left(H_{1}, H_{2}, H_{2}\right), g\left(H_{1}, H_{1}, H_{2}\right)$ and $g\left(H_{1}, H_{1}, H_{1}\right)$. 

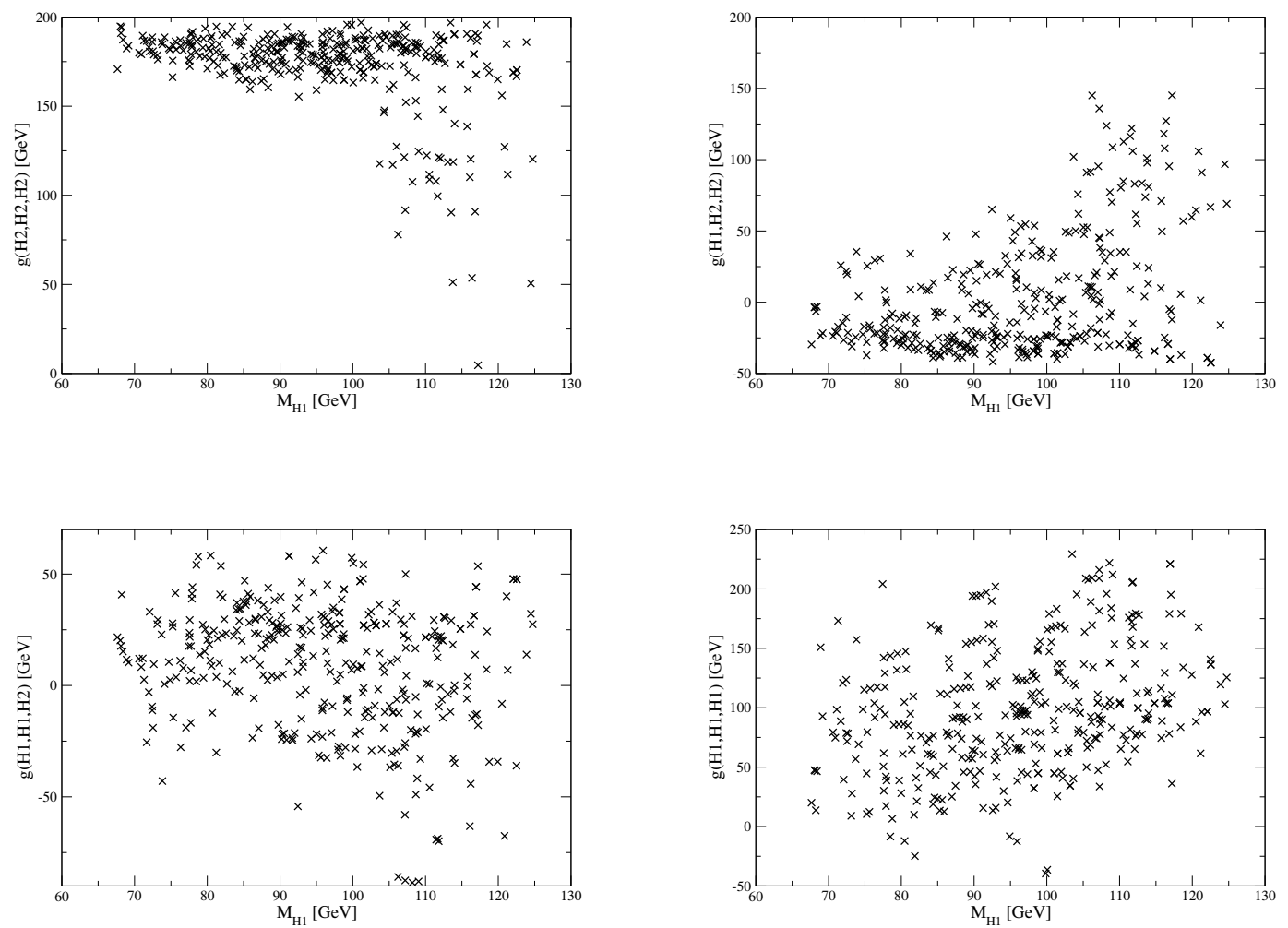

Figure 1. Scatter plots of the trilinear Higgs couplings $g\left(H_{2}, H_{2}, H_{2}\right)$ (top left), $g\left(H_{1}, H_{2}, H_{2}\right)$ (top right), $g\left(H_{1}, H_{1}, H_{2}\right)$ (bottom left) and $g\left(H_{1}, H_{1}, H_{1}\right)$ (bottom right) as function of $M_{H_{1}}$.

We see that the trilinear coupling of the SM-like state $H_{2}$ (top left) is typically close to its SM value, except for a possible reduction if $M_{H_{1}} \gtrsim 100 \mathrm{GeV}$. The couplings $g\left(H_{1}, H_{2}, H_{2}\right)$ and $g\left(H_{1}, H_{1}, H_{2}\right)$ are smaller, just $g\left(H_{1}, H_{2}, H_{2}\right)$ can assume similar values as $g\left(H_{2}, H_{2}, H_{2}\right)$ for $M_{H_{1}} \gtrsim 100 \mathrm{GeV}$. The coupling $g\left(H_{1}, H_{1}, H_{1}\right)$ can vary from small up to SM-like values, with no specific dependence on $M_{H_{1}}$. Values significantly larger than in the SM are not observed.

Next we turn to the Higgs pair production cross sections in gluon fusion at the LHC at $14 \mathrm{TeV}$. As stated in the Introduction, we make the conservative assumptions that neither light stops nor the heavy state $H_{3}$ contribute to the cross sections. Since observations of Higgs pair production will certainly require large integrated luminosities of at least 300$600 \mathrm{fb}^{-1}$, these particles should otherwise have been observed by then in which case the present calculations can be correspondingly corrected. Besides the triple Higgs couplings shown before, also the Higgs top couplings are relevant here.

We normalize all Higgs pair production cross sections to the SM value of about $32.6 \mathrm{fb}$ from HPAIR. First, this value will certainly be used as "benchmark" by the experimental collaborations in order to test the trilinear Higgs coupling of the SM. Second, one can expect that this ratio should remain approximately invariant under higher order QCD cor- 

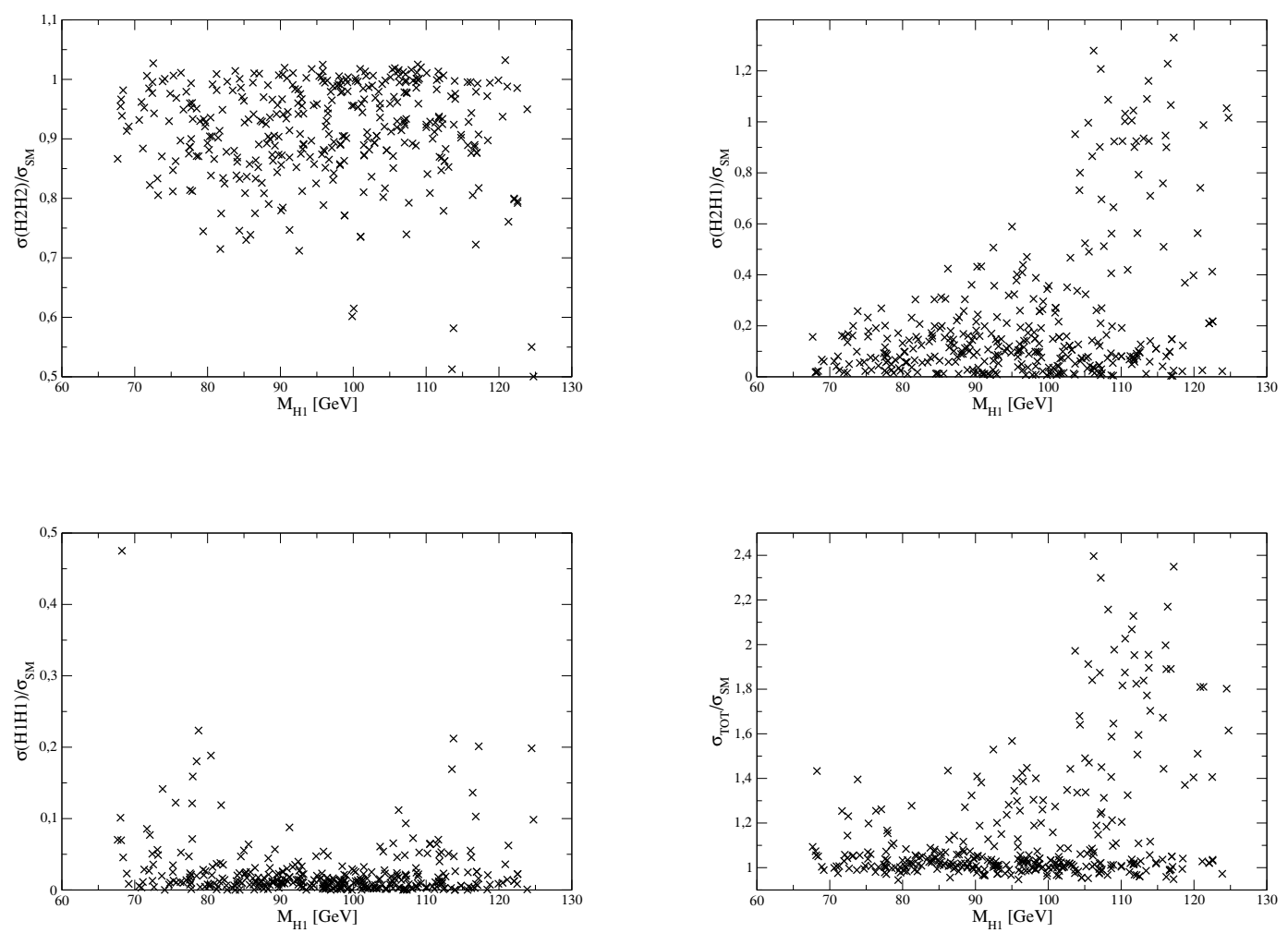

Figure 2. The Higgs pair production cross sections relative to the SM for the final states $H_{2}+H_{2}$ (top left), $H_{2}+H_{1}$ (top right), $H_{1}+H_{1}$ (bottom left) and the sum over all final states (bottom right).

rections and the replacement of the heavy top quark limit in HPAIR by the full matrix element, although the latter may be relevant for specific simulations [19]. In figure 2 we show the normalized Higgs pair production cross sections for the final states $\mathrm{H}_{2}+\mathrm{H}_{2}$, $H_{2}+H_{1}, H_{1}+H_{1}$ and the sum over all final states.

In figure 2 we see no significant enhancement of the cross section for the final state $H_{2}+H_{2}$ (in agreement with the results obtained in [26] for heavy stops), a possible enhancement for the final state $H_{2}+H_{1}$ for $M_{H_{1}} \gtrsim 100 \mathrm{GeV}$, and always below-the-SM values for the final state $H_{1}+H_{1}$. Unfortunately there exist regions in the parameter space where the cross sections for both final states $H_{2}+H_{1}$ and $H_{1}+H_{1}$ are small. Since $H_{1}$ has typically reduced couplings to top quarks, its production has to rely essentially on a virtual mostly SM-like $\mathrm{H}_{2}$ in the s-channel. However, the then relevant trilinear couplings $g\left(H_{1}, H_{2}, H_{2}\right)$ and $g\left(H_{1}, H_{1}, H_{2}\right)$ can simultaneously be small leading to a poor production rate for both $H_{2}+H_{1}$ and $H_{1}+H_{1}$ final states, which are relevant for the $H_{1}$ discovery. The good news is, on the other hand, that the sum over all final states is never below the $\mathrm{SM}$ value, but up to $\sim 2.5$ times larger for $M_{H_{1}} \gtrsim 100 \mathrm{GeV}$.

However, Higgs pair production will only be measurable in specific channels. First we consider the $b \bar{b}+\tau^{+} \tau^{-}$final state which has been studied in [19]. Since the branching fractions of both $H_{1}$ and $H_{2}$ can differ significantly from a SM-like Higgs boson (limited some- 

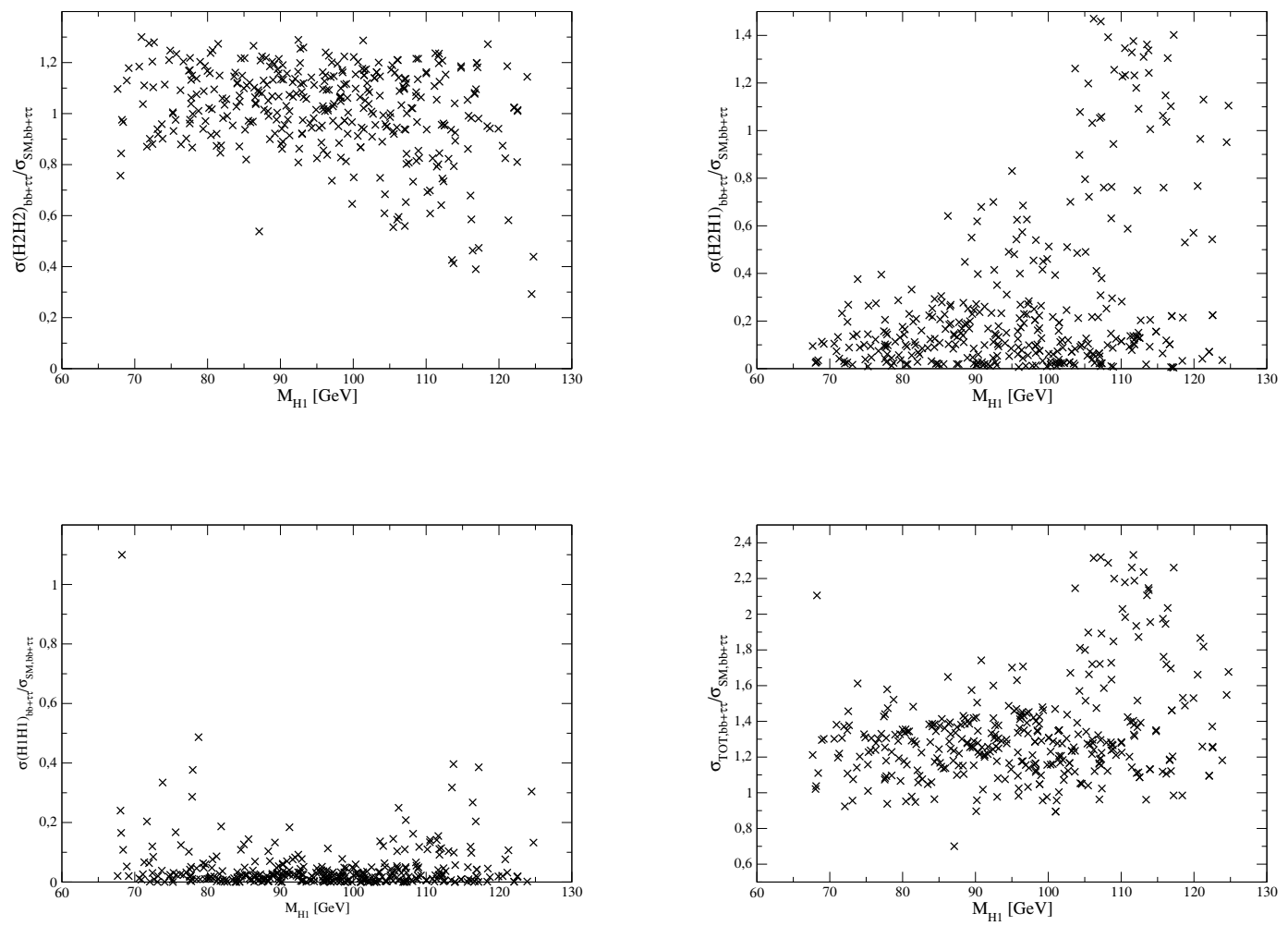

Figure 3. The Higgs pair production cross sections relative to the SM for the final states $H_{2}+H_{2} \rightarrow$ $b \bar{b}+\tau^{+} \tau^{-}$(top left), $H_{2}+H_{1} \rightarrow b \bar{b}+\tau^{+} \tau^{-}$(top right), $H_{1}+H_{1} \rightarrow b \bar{b}+\tau^{+} \tau^{-}$(bottom left) and the sum over all $H_{i}$ into $b \bar{b}+\tau^{+} \tau^{-}$(bottom right).

what, in the case of $\mathrm{H}_{2}$, by the combinations of the LHC and Tevatron measurements) one should weight the production cross sections by the corresponding branching fractions relative to the SM. In the NMSSM for low $\tan \beta$ one can assume that the relative variations of the branching fractions into $b \bar{b}$ and $\tau^{+} \tau^{-}$are practically the same, since both couplings originate from the $H_{d}$ component of $H_{1}$ and $H_{2}$. Hence, in the case of $H_{2}+H_{1}$, these cross sections weighted by the branching fractions will be the same for $H_{2}$ or $H_{1}$ decaying into $\tau^{+} \tau^{-}$.

In figure 3 we show the normalized Higgs pair production cross sections for the final states $H_{2}+H_{2} \rightarrow b \bar{b}+\tau^{+} \tau^{-}, H_{2}+H_{1} \rightarrow b \bar{b}+\tau^{+} \tau^{-}, H_{1}+H_{1} \rightarrow b \bar{b}+\tau^{+} \tau^{-}$and the sum over all $H_{i}$ into $b \bar{b}+\tau^{+} \tau^{-}$.

Although the cross sections weighted by the branching fractions relative to the SM differ in general from the full relative cross sections shown in figure 2 for given points in parameter space, the general pattern is similar: the sum over cross sections for all Higgs pairs is practically never below the SM value, and can be amplified by a factor $\sim 2.4$. This originates from possible $H_{2}+H_{1} \rightarrow b \bar{b}+\tau^{+} \tau^{-}$processes, whose cross sections can reach beyond-the-SM values by itself.

Unfortunately the production of the lighter Higgs boson $H_{1}$ in either the $H_{2}+H_{1} \rightarrow$ $b \bar{b}+\tau^{+} \tau^{-}$or $H_{1}+H_{1} \rightarrow b \bar{b}+\tau^{+} \tau^{-}$final state does not always have a cross section of 

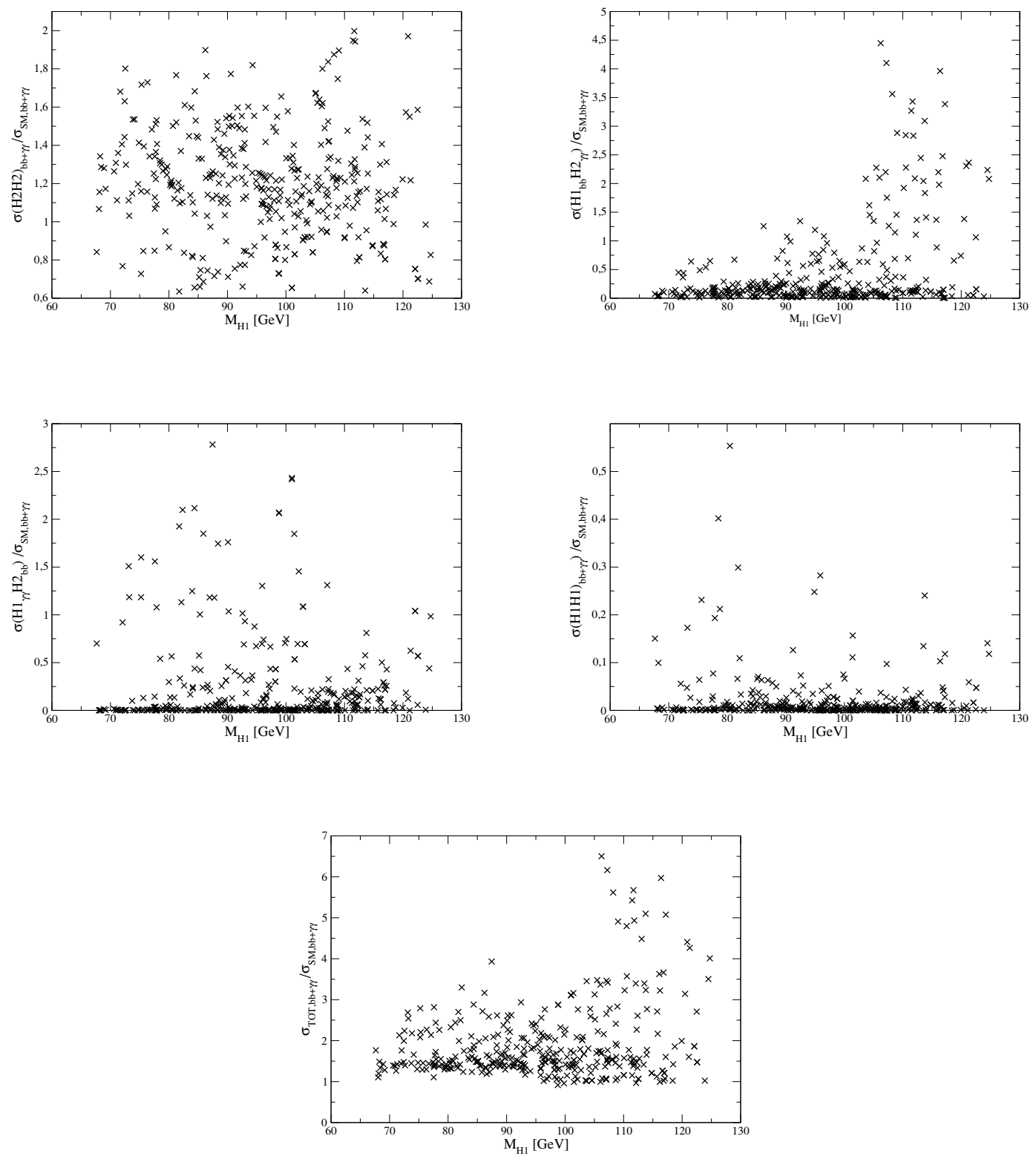

Figure 4. The Higgs pair production cross sections relative to the SM for the final states $H_{2}+H_{2} \rightarrow$ $b \bar{b}+\gamma \gamma$ (top left), $\left(H_{1} \rightarrow b \bar{b}\right)+\left(H_{2} \rightarrow \gamma \gamma\right)$ (top right), $\left(H_{1} \rightarrow \gamma \gamma\right)+\left(H_{2} \rightarrow b \bar{b}\right)$ (middle left), $H_{1}+H_{1} \rightarrow b \bar{b}+\gamma \gamma$ (middle right) and the sum over all $H_{i}$ into $b \bar{b}+\gamma \gamma$ (bottom).

the order the SM Higgs pair production. Hence the detection of $H_{1}$ in this channel is a spectacular possibility, but not guaranteed.

Another potential Higgs pair discovery channel is the $b \bar{b}+\gamma \gamma$ final state [17]. Again we consider the production cross sections multiplied by the branching fractions relative to the ones of a $125 \mathrm{GeV}$ SM-like Higgs boson, which would be the target once such searches will be performed. In this case the final states $\left(H_{1} \rightarrow b \bar{b}\right)+\left(H_{2} \rightarrow \gamma \gamma\right)$ and $\left(H_{2} \rightarrow b \bar{b}\right)+\left(H_{1} \rightarrow \gamma \gamma\right)$ 
have to be considered separately. The relative cross sections times branching fractions for all possible distinct final states together with their sum are shown in figure 4 .

In figure 4 we see cases of spectacular enhancements with respect to the SM cross section in this channel. In the final state $\left(H_{1} \rightarrow b \bar{b}\right)+\left(H_{2} \rightarrow \gamma \gamma\right.$ ) (up to a factor $\sim 4.5$, top right) these originate from an enhancement of the branching fraction for $H_{2} \rightarrow \gamma \gamma$ by a factor up to $\sim 2.3$, consistent with the present measurements of signal rates at the LHC due to a slightly reduced $\mathrm{H}_{2}$ production cross section, together with enhancements of the branching fraction for $H_{1} \rightarrow b \bar{b}$ and the production cross section into $H_{2}+H_{1}$. Due to the absence of the latter factors and a reduction of the branching fraction for $H_{2} \rightarrow b \bar{b}$, the enhancements of the cross sections in the final state $H_{2}+H_{2} \rightarrow b \bar{b}+\gamma \gamma$ for the same points in the top left of figure 4 are less spectacular, but lead to relative signal rates up to a factor $\sim 6.5$ larger for the sum over the final states $H_{1}+H_{2}$ and $H_{2}+H_{2}$. (These final states differ, however, in the invariant mass of the $b \bar{b}$ system corresponding to $M_{H_{1}}$ or $M_{H_{2}}$, respectively.)

Also in the distinguishable final state $\left(H_{1} \rightarrow \gamma \gamma\right)+\left(H_{2} \rightarrow b \bar{b}\right)$ enhancements up to a factor $\sim 2.8$ are possible. These originate from enhancements of the branching fraction for $H_{1} \rightarrow \gamma \gamma$, a possibility pointed out in [61]. On the other hand, as before it is not guaranteed that any cross section involving $H_{1}$ is measurably large; one just ends up with a "no-lose theorem" stating that at least the cross section for the sum over all $H_{1}, H_{2}$ final states never falls below the SM cross section.

\section{Conclusions}

The regions in the NMSSM parameter space giving rise to another mostly singlet-like Higgs boson $H_{1}$ with a mass below $125 \mathrm{GeV}$ are particularly natural, since here mixing effects lead to an increase of the mass of the mostly SM-like state $H_{2}$. In the present work we studied the possible impact of such mixings on the Higgs pair production cross sections in gluon fusion at $14 \mathrm{TeV}$ and the $b \bar{b}+\tau^{+} \tau^{-}$and $b \bar{b}+\gamma \gamma$ final states. A priori it was not clear whether, even after summing over the $H_{1}, H_{2}$ final states, the cross sections are always as large as in the SM for a single $125 \mathrm{GeV}$ Higgs boson. Our results indicate that this is the case. Cross sections larger than in the SM are possible, notably in the $b \bar{b}+\gamma \gamma$ final state involving both $H_{1}$ and $H_{2}$. But unfortunately it is not guaranteed that the additional state $H_{1}$ has always a sufficiently large production rate in Higgs pair production to be detectable; in this case one would have to rely on either its direct production, or on decays of the heavier state $H_{3}$ into $H_{1}$.

On the other hand, given the possibility that $H_{1}$ is visible only in Higgs pair production processes, sufficiently flexible cuts should be applied in future analyses in order not to miss it even for $M_{H_{1}}$ well below $125 \mathrm{GeV}$.

\section{Acknowledgments}

We acknowledge support from the French ANR LFV-CPV-LHC, ANR STR-COSMO, the European Union FP7 ITN INVISIBLES (Marie Curie Actions, PITN-GA-2011-289442) and the ERC advanced grant Higgs@LHC. 
Open Access. This article is distributed under the terms of the Creative Commons Attribution License which permits any use, distribution and reproduction in any medium, provided the original author(s) and source are credited.

\section{References}

[1] ATLAS collaboration, Combined coupling measurements of the Higgs-like boson with the ATLAS detector using up to $25 \mathrm{fb}^{-1}$ of proton-proton collision data, ATLAS-CONF-2013-034, CERN, Geneva Switzerland (2013).

[2] CMS collaboration, Combination of Standard Model Higgs boson searches and measurements of the properties of the new boson with a mass near $125 \mathrm{GeV}$, CMS-PAS-HIG-13-005, CERN, Geneva Switzerland (2013).

[3] O.J. Eboli, G. Marques, S. Novaes and A. Natale, Twin Higgs boson production, Phys. Lett. B 197 (1987) 269 [INSPIRE].

[4] D.A. Dicus, C. Kao and S.S. Willenbrock, Higgs boson pair production from gluon fusion, Phys. Lett. B 203 (1988) 457 [inSPIRE].

[5] E.N. Glover and J. van der Bij, Higgs boson pair production via gluon fusion, Nucl. Phys. B 309 (1988) 282 [inSPIRE].

[6] T. Plehn, M. Spira and P. Zerwas, Pair production of neutral Higgs particles in gluon-gluon collisions, Nucl. Phys. B 479 (1996) 46 [Erratum ibid. B 531 (1998) 655] [hep-ph/9603205] [INSPIRE].

[7] S. Dawson, S. Dittmaier and M. Spira, Neutral Higgs boson pair production at hadron colliders: QCD corrections, Phys. Rev. D 58 (1998) 115012 [hep-ph/9805244] [INSPIRE].

[8] A. Djouadi, W. Kilian, M. Muhlleitner and P. Zerwas, Production of neutral Higgs boson pairs at LHC, Eur. Phys. J. C 10 (1999) 45 [hep-ph/9904287] [INSPIRE].

[9] A. Belyaev, M. Drees, O.J. Eboli, J. Mizukoshi and S. Novaes, Supersymmetric Higgs pair production at hadron colliders, Phys. Rev. D 60 (1999) 075008 [hep-ph/9905266] [INSPIRE].

[10] A. Belyaev, M. Drees and J. Mizukoshi, Supersymmetric Higgs boson pair production: discovery prospects at hadron colliders, Eur. Phys. J. C 17 (2000) 337 [hep-ph/9909386] [INSPIRE].

[11] G. Cynolter, E. Lendvai and G. Pocsik, Resonance production of three neutral supersymmetric Higgs bosons at LHC, Acta Phys. Polon. B 31 (2000) 1749 [hep-ph/0003008] [INSPIRE].

[12] C. Kim, K.Y. Lee and J.-H. Song, Enhancement of the Higgs pair production at CERN LHC: the MSSM and extra dimension effects, Phys. Rev. D 64 (2001) 015009 [hep-ph/0009231] [INSPIRE].

[13] A. Barrientos Bendezu and B.A. Kniehl, Pair production of neutral Higgs bosons at the CERN Large Hadron Collider, Phys. Rev. D 64 (2001) 035006 [hep-ph/0103018] [INSPIRE].

[14] U. Baur, T. Plehn and D.L. Rainwater, Measuring the Higgs boson self coupling at the LHC and finite top mass matrix elements, Phys. Rev. Lett. 89 (2002) 151801 [hep-ph/0206024] [INSPIRE].

[15] U. Baur, T. Plehn and D.L. Rainwater, Determining the Higgs boson selfcoupling at hadron colliders, Phys. Rev. D 67 (2003) 033003 [hep-ph/0211224] [InSPIRE]. 
[16] U. Baur, T. Plehn and D.L. Rainwater, Examining the Higgs boson potential at lepton and hadron colliders: a comparative analysis, Phys. Rev. D 68 (2003) 033001 [hep-ph/0304015] [INSPIRE].

[17] U. Baur, T. Plehn and D.L. Rainwater, Probing the Higgs selfcoupling at hadron colliders using rare decays, Phys. Rev. D 69 (2004) 053004 [hep-ph/0310056] [INSPIRE].

[18] T. Binoth, S. Karg, N. Kauer and R. Ruckl, Multi-Higgs boson production in the Standard Model and beyond, Phys. Rev. D 74 (2006) 113008 [hep-ph/0608057] [INSPIRE].

[19] M.J. Dolan, C. Englert and M. Spannowsky, Higgs self-coupling measurements at the LHC, JHEP 10 (2012) 112 [arXiv:1206.5001] [INSPIRE].

[20] N.D. Christensen, T. Han and T. Li, Pair production of MSSM Higgs bosons in the non-decoupling region at the LHC, Phys. Rev. D 86 (2012) 074003 [arXiv:1206.5816] [INSPIRE].

[21] A. Papaefstathiou, L.L. Yang and J. Zurita, Higgs boson pair production at the LHC in the $b \bar{b} W^{+} W^{-}$channel, Phys. Rev. D 87 (2013) 011301 [arXiv:1209.1489] [InSPIRE].

[22] M.J. Dolan, C. Englert and M. Spannowsky, New physics in LHC Higgs boson pair production, Phys. Rev. D 87 (2013) 055002 [arXiv:1210.8166] [INSPIRE].

[23] J. Baglio et al., The measurement of the Higgs self-coupling at the LHC: theoretical status, JHEP 04 (2013) 151 [arXiv:1212.5581] [INSPIRE].

[24] D.Y. Shao, C.S. Li, H.T. Li and J. Wang, Threshold resummation effects in Higgs boson pair production at the LHC, arXiv:1301.1245 [INSPIRE].

[25] F. Goertz, A. Papaefstathiou, L.L. Yang and J. Zurita, Higgs boson self-coupling measurements using ratios of cross sections, JHEP 06 (2013) 016 [arXiv:1301.3492] [INSPIRE].

[26] J. Cao, Z. Heng, L. Shang, P. Wan and J.M. Yang, Pair production of a $125 \mathrm{GeV}$ Higgs boson in MSSM and NMSSM at the LHC, JHEP 04 (2013) 134 [arXiv:1301.6437] [INSPIRE].

[27] J. Grigo, J. Hoff, K. Melnikov and M. Steinhauser, On the Higgs boson pair production at the $L H C$, arXiv: 1305.7340 [INSPIRE].

[28] D.T. Nhung, M. Muhlleitner, J. Streicher and K. Walz, Higher order corrections to the trilinear Higgs self-couplings in the real NMSSM, arXiv:1306.3926 [INSPIRE].

[29] U. Ellwanger, C. Hugonie and A.M. Teixeira, The next-to-minimal supersymmetric Standard Model, Phys. Rept. 496 (2010) 1 [arXiv:0910.1785] [INSPIRE].

[30] L.J. Hall, D. Pinner and J.T. Ruderman, A natural SUSY Higgs near $126 \mathrm{GeV}$, JHEP 04 (2012) 131 [arXiv:1112.2703] [INSPIRE].

[31] U. Ellwanger, A Higgs boson near $125 \mathrm{GeV}$ with enhanced di-photon signal in the NMSSM, JHEP 03 (2012) 044 [arXiv: 1112.3548] [INSPIRE].

[32] A. Arvanitaki and G. Villadoro, A non Standard Model Higgs at the LHC as a sign of naturalness, JHEP 02 (2012) 144 [arXiv:1112.4835] [INSPIRE].

[33] S. King, M. Muhlleitner and R. Nevzorov, NMSSM Higgs benchmarks near $125 \mathrm{GeV}$, Nucl. Phys. B 860 (2012) 207 [arXiv:1201.2671] [INSPIRE].

[34] Z. Kang, J. Li and T. Li, On naturalness of the MSSM and NMSSM, JHEP 11 (2012) 024 [arXiv: 1201.5305] [INSPIRE]. 
[35] J.-J. Cao, Z.-X. Heng, J.M. Yang, Y.-M. Zhang and J.-Y. Zhu, A SM-like Higgs near $125 \mathrm{GeV}$ in low energy SUSY: a comparative study for MSSM and NMSSM, JHEP 03 (2012) 086 [arXiv: 1202.5821] [INSPIRE].

[36] U. Ellwanger and C. Hugonie, Higgs bosons near $125 \mathrm{GeV}$ in the NMSSM with constraints at the GUT scale, Adv. High Energy Phys. 2012 (2012) 625389 [arXiv:1203.5048] [INSPIRE].

[37] K.S. Jeong, Y. Shoji and M. Yamaguchi, Singlet-doublet Higgs mixing and its implications on the Higgs mass in the PQ-NMSSM, JHEP 09 (2012) 007 [arXiv:1205.2486] [INSPIRE].

[38] L. Randall and M. Reece, Single-scale natural SUSY, arXiv:1206.6540 [INSPIRE].

[39] R. Benbrik et al., Confronting the MSSM and the NMSSM with the discovery of a signal in the two photon channel at the LHC, Eur. Phys. J. C 72 (2012) 2171 [arXiv:1207.1096] [INSPIRE].

[40] B. Kyae and J.-C. Park, A singlet-extension of the MSSM for $125 \mathrm{GeV}$ Higgs with the least tuning, Phys. Rev. D 87 (2013) 075021 [arXiv: 1207.3126] [INSPIRE].

[41] J. Cao, Z. Heng, J.M. Yang and J. Zhu, Status of low energy SUSY models confronted with the LHC $125 \mathrm{GeV}$ Higgs data, JHEP 10 (2012) 079 [arXiv:1207.3698] [inSPIRE].

[42] K. Agashe, Y. Cui and R. Franceschini, Natural islands for a $125 \mathrm{GeV}$ Higgs in the scale-invariant NMSSM, JHEP 02 (2013) 031 [arXiv:1209.2115] [INSPIRE].

[43] G. Bélanger et al., Higgs bosons at 98 and $125 \mathrm{GeV}$ at LEP and the LHC, JHEP 01 (2013) 069 [arXiv:1210.1976] [INSPIRE].

[44] Z. Heng, A $125 \mathrm{GeV}$ Higgs and its di-photon signal in different SUSY models: a mini review, arXiv: 1210.3751 [INSPIRE].

[45] K. Choi, S.H. Im, K.S. Jeong and M. Yamaguchi, Higgs mixing and diphoton rate enhancement in NMSSM models, JHEP 02 (2013) 090 [arXiv:1211.0875] [INSPIRE].

[46] S. King, M. Mühlleitner, R. Nevzorov and K. Walz, Natural NMSSM Higgs bosons, Nucl. Phys. B 870 (2013) 323 [arXiv:1211.5074] [InSPIRE].

[47] T. Gherghetta, B. von Harling, A.D. Medina and M.A. Schmidt, The scale-invariant NMSSM and the $126 \mathrm{GeV}$ Higgs boson, JHEP 02 (2013) 032 [arXiv:1212.5243] [INSPIRE].

[48] T. Cheng, J. Li, T. Li and Q.-S. Yan, Natural NMSSM confronting with the LHC7-8, arXiv:1304.3182 [INSPIRE].

[49] R. Barbieri, D. Buttazzo, K. Kannike, F. Sala and A. Tesi, Exploring the Higgs sector of a most natural NMSSM, Phys. Rev. D 87 (2013) 115018 [arXiv:1304.3670] [INSPIRE].

[50] M. Badziak, M. Olechowski and S. Pokorski, New regions in the NMSSM with a $125 \mathrm{GeV}$ Higgs, JHEP 06 (2013) 043 [arXiv: 1304.5437] [INSPIRE].

[51] T. Cheng and T. Li, Electroweak supersymmetry (EWSUSY) in the NMSSM, arXiv:1305.3214 [INSPIRE].

[52] E. Hardy, Is natural SUSY natural?, arXiv:1306.1534 [INSPIRE].

[53] ALEPH, DELPHI, L3, OPAL and LEP Working Group for Higgs Boson Searches collaborations, S. Schael et al., Search for neutral MSSM Higgs bosons at LEP, Eur. Phys. J. C 47 (2006) 547 [hep-ex/0602042] [INSPIRE].

[54] R.S. Gupta, H. Rzehak and J.D. Wells, How well do we need to measure the Higgs boson mass and self-coupling?, arXiv:1305.6397 [INSPIRE]. 
[55] Z. Kang, J. Li, T. Li, D. Liu and J. Shu, Probing the CP-even Higgs sector via $\mathrm{H}_{3} \rightarrow \mathrm{H}_{2} \mathrm{H}_{1}$ in the natural NMSSM, arXiv:1301.0453 [INSPIRE].

[56] G. Bélanger, B. Dumont, U. Ellwanger, J. Gunion and S. Kraml, Global fit to Higgs signal strengths and couplings and implications for extended Higgs sectors, arXiv:1306.2941 [INSPIRE].

[57] U. Ellwanger, J.F. Gunion and C. Hugonie, NMHDECAY: a Fortran code for the Higgs masses, couplings and decay widths in the NMSSM, JHEP 02 (2005) 066 [hep-ph/0406215] [INSPIRE].

[58] U. Ellwanger and C. Hugonie, NMHDECAY 2.0: an updated program for sparticle masses, Higgs masses, couplings and decay widths in the NMSSM, Comput. Phys. Commun. 175 (2006) 290 [hep-ph/0508022] [INSPIRE].

[59] NMSSMTools: tools for the calculation of the Higgs and sparticle spectrum in the NMSSM webpage, http://www.th.u-psud.fr/NMHDECAY/nmssmtools.html.

[60] M. Spira, Personal web page http://people.web.psi.ch/spira/hpair.

[61] U. Ellwanger, Enhanced di-photon Higgs signal in the next-to-minimal supersymmetric Standard Model, Phys. Lett. B 698 (2011) 293 [arXiv:1012.1201] [INSPIRE]. 